\title{
NUMERICAL VERIFICATION OF VELOCITY DISTRIBUTION MODELS IN A SELECTED VENTILATION DUCT
}

\author{
Peszyński K.*, Mrozik D.**
}

\begin{abstract}
Paper presents a comparative analysis of four, previously developed, theoretical models of air velocity distribution in the selected ventilation duct with the results of calculations carried out in the ANSYSFLUENT code. The results of numerical tests related to the velocity profile to its full development are presented. Dimensionless flow rate values resulting from the developed models were also referenced to the flow rate used during simulation.
\end{abstract}

Keywords: Turbulent flow, Simulation, Ventilation duct, Power-law velocity profile, ANSYS-FLUENT.

\section{Introduction}

During the development of a new ventilation system based on ducts with a rounded rectangular crosssection, a large number of experimental tests were performed. The purpose of this research was to determine linear (major) losses (Peszyński, 2019) in ducts and local (minor) losses (Smyk et al., 2017) in system components.

The main problem during the tests was the accurate determination of the volumetric flow rate in all 79 crosssections examined. Due to the unusual shape of the ducts, it was not possible to use popular methods for determining the average volume flow rate (e.g. orifice, flow nozzle, and venturi obstruction meters) because it would be necessary to make additional 158 transition elements matching the cross-section of the rounded rectangle to the circular cross-section. This problem was avoided by measuring the volumetric flow rate at the inlet to the supply fan. In view of the above, the volumetric flow rate was determined on the basis of derived mathematical models based on the velocity distribution along two perpendicular symmetry axes of the cross-section. A total of 4 models of volumetric flow rate determination were developed (Peszyński, 2018).

2. Development of the velocity profile along the length location of the ventilation duct

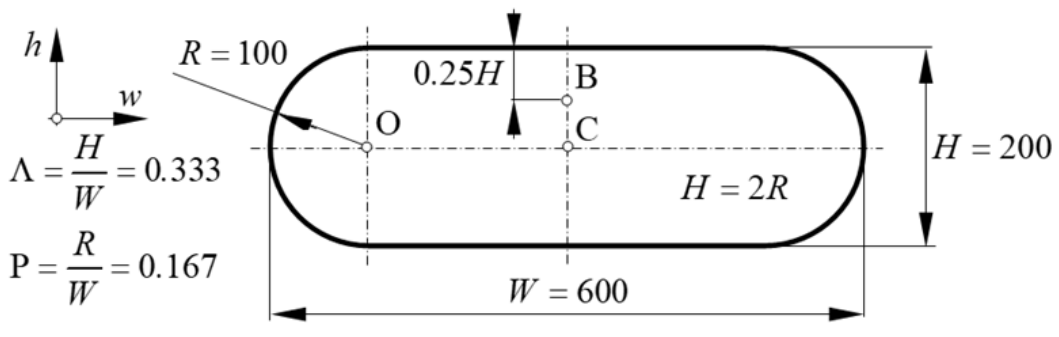

$$
\begin{aligned}
A_{\mathrm{rr}} & =W H-(4-\pi) R^{2} \\
& =0.6 \cdot 0.2-(4-\pi) 0.1^{2}=0.111 \mathrm{~m}^{2} \\
U_{\mathrm{rr}} & =2(W-H+\pi R) \\
& =2(0.6-0.2+\pi 0.1)=1.482 \mathrm{~m} \\
D_{\mathrm{H}} & =\frac{4 A_{\mathrm{rr}}}{U_{\mathrm{rr}}}=0.312 \mathrm{~m}
\end{aligned}
$$

Fig. 1: Cross-section of the examined duct and its basic parameters.

The duct with a rounded rectangular cross-section begins with its downstream assembly (Peszyński, 2018) into the mask of the tubular air deflector. From 79 different cross-sections examined for analysis in this article, the one shown in Fig. 1 was selected. The examined duct consisted of 12 segments with a length of $1.5 \mathrm{~m}$ each, therefore it was $L=18 \mathrm{~m}$ in total.

* Assoc. Prof. Kazimierz Peszyński, PhD.: UTP University of Science and Technology, Faculty of Mechanical Engineering Al. Prof. S. Kaliskiego 7, Bydgoszcz; PL, kazimierz.peszynski@utp.edu.pl

** Dariusz Mrozik, MSc.: UTP University of Science and Technology, Faculty of Mechanical Engineering Al. Prof. S. Kaliskiego 7, Bydgoszcz; PL, dariuszmrozik1@gmail.com 
The flow in this duct was modeled using the ANSYS-FLUENT code. During simulation turbulence model $k-\omega$ was used. The CFD calculation mesh was dependent on the turbulence model and flow parameters selected (Abe et al., 2001), near the walls was used refinement - see Fig. 2, created on the nondimensional parameter $y^{+}$. For $y^{+}=1$ based on viscous velocity an kinetic viscosity the thickness of the boundary layer $y=0.13 \mathrm{~mm}$ was determined.

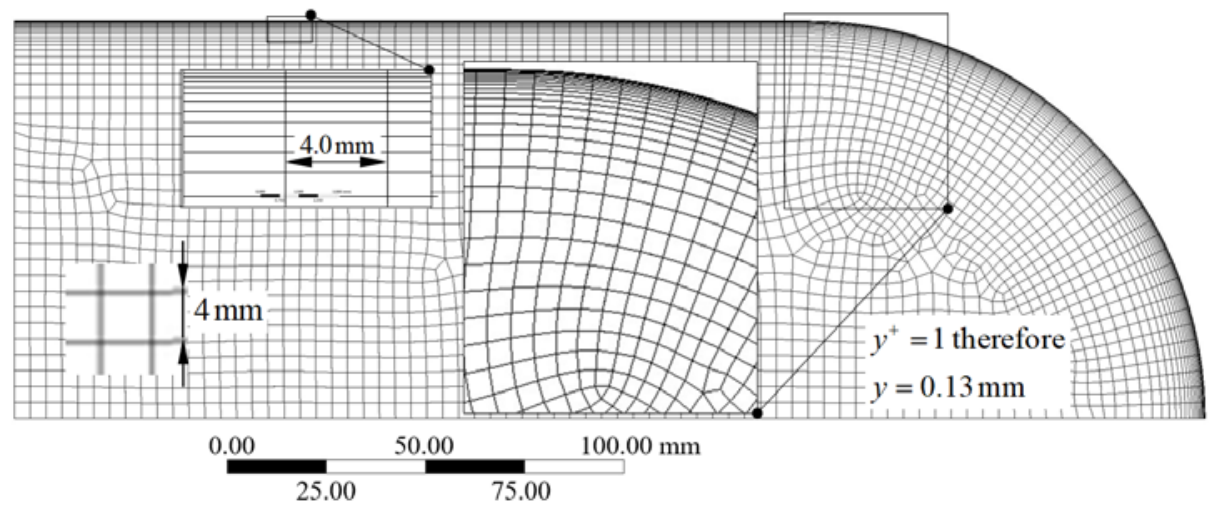

Fig. 2: Mesh with refinement near the wall.

The $0.5 \mathrm{~m}$ long model had nearly 500000 finite elements. For the rest of the duct length, the grid was not corrected. Such a model had only about 250000 finite elements.

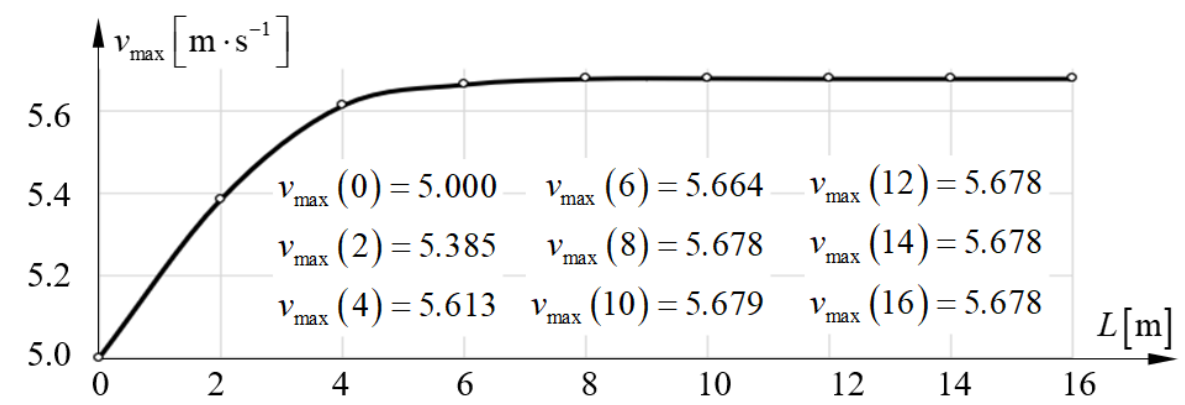

Fig. 3: Computed maximum velocity line on the examined duct axis.

At the entrance to the duct a rectangular velocity profile $v(w, h)=v_{\text {arg }}=5 \mathrm{~m} \cdot \mathrm{s}^{-1}$ was adopted, than flow rate is $\dot{V}=0.557 \mathrm{~m}^{2}$. During experimental research, it was assumed that the minimum length of the duct necessary to stabilize changes in flow velocity on its axis should be $L=20 D_{\mathrm{h}}$, which on the basis of Fig. 1 gives the value of $L=6.24 \mathrm{~m}$. From Fig. 3 we see, that $v(6) / v(16)=0.9993$, it means that the velocity is stabilized, which confirms the assumption made during the measurements.

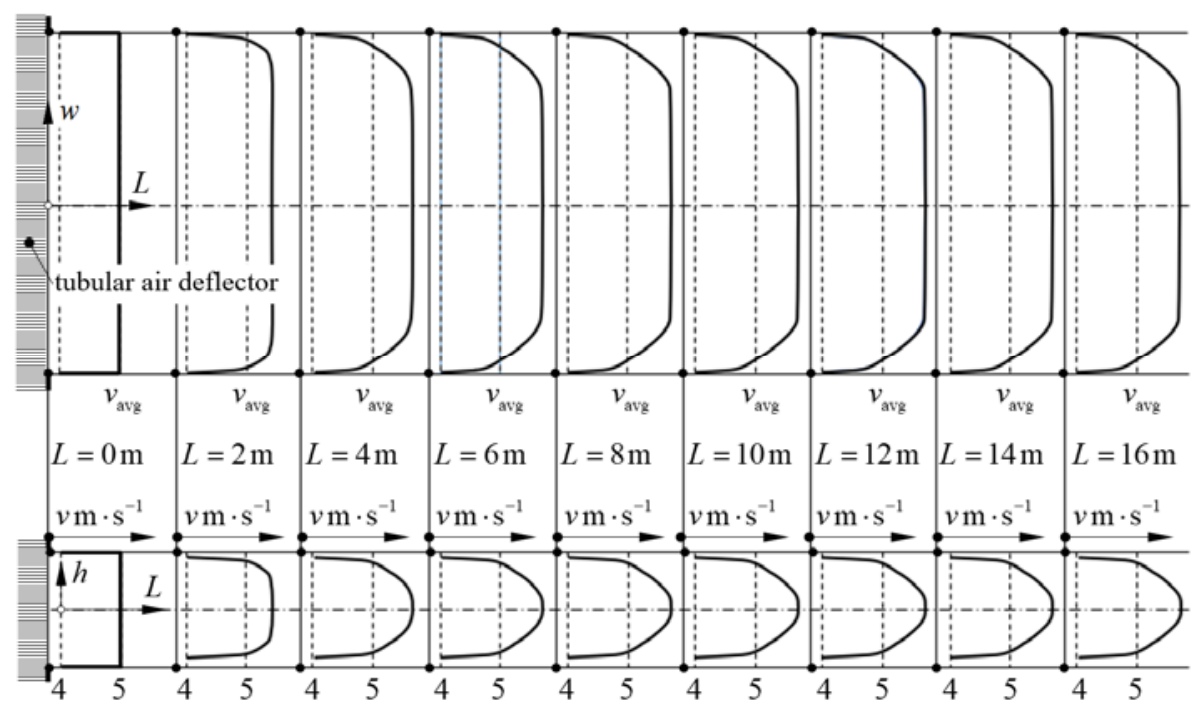

Fig. 4: Development of velocity profile along duct axis. 
During the development of velocity distribution models in cross-sections, it was assumed that the velocity distribution along the long axis is practically constant over a certain it part, symmetrical to duct axis $L$, Fig. 4a confirms this assumption. Another assumption was that the side parts of the velocity distribution along the long axis are similar to the velocity distribution along the short one, Fig $4 \mathrm{~b}$ confirms this assumption.

\section{Verification of velocity distribution in models}

\subsection{Basic power-law velocity profile in uniform cross-section}

In this model, it is assumed that the velocity distribution over the entire cross-sectional area of the duct is deter-mined by the same formula, known as power-law velocity profile. The adjective "uniform" has been added in the title of this subsection, because in subsection 3.2 the cross-section divided into two parts will be analyzed. Average velocity is determined by formula (Peszyński, 2018)

$$
\dot{\mathrm{V}}_{\mathrm{rr}}=v_{\mathrm{arg}} A_{\mathrm{rr}}=\frac{n}{n+1}\left(\frac{n}{n+1} \Lambda\left(1-\left(\frac{4 \mathrm{P}^{2}}{\Lambda}\right)^{\frac{n+1}{n}}\right)+\pi\left(\frac{4 \mathrm{P}^{2}}{\Lambda}\right)^{\frac{1}{n}} \frac{2 n \mathrm{P}^{2}}{2 n+1}\right) W^{2} v_{\mathrm{C}}
$$

Note, that term before $W^{2} v_{\mathrm{C}}$ is dimensionless, it contains only dimensionless construction parameters and the denominator $n$ of the exponent found in the power law velocity profile. Two cases will be considered: (a) $n=7$ - coefficient considered by a significant number of scientists to be accurate enough to describe the turbulent air flow, and (b) $n=n_{\text {opt }}$ obtained by optimally matching the denominator $n$ to the velocity profile determined during the simulation. The optimization consisted of creating a goal function $\Delta$ in the form of a sum of squares of differences between the value $v_{\text {si }}$ obtained during the simulation and the value $v_{\mathrm{o} i}$ resulting from the approximation using power law velocity profile $\left(v_{\mathrm{s} i}-v_{\mathrm{o} i}\right)^{2}$, therefore the goal function has the form

$$
\Delta=\sum_{i=1}^{51}\left(v_{\mathrm{si}}-v_{\mathrm{o} i}\right)^{2} \Rightarrow \Delta_{\min }\left(n, v_{\mathrm{C}}\right)
$$

Dimension $H$ has been divided into 50 sections with a length of $\Delta H_{i}=4 \mathrm{~mm}$ each, which gives 51 velocity reading points. The function (2) was minimized using Solver a Microsoft Excel add-in program. In the calculations below, the volume flow through the duct $\dot{V}_{\text {rrs }}=0.557 \mathrm{~m}^{3} \cdot \mathrm{s}^{-1}$ was taken as the reference value.

1. Using simulation result $v_{\mathrm{C} 16}=5.678 \mathrm{~m} \cdot \mathrm{s}^{-1}, n=7$, and parameters $\Lambda=0.333, \mathrm{P}=0.167, W=0.6 \mathrm{~m}$ (see Fig. 1) after substitution to formula (1), we obtain $\dot{V}_{\text {rr nn=7 }}=0.497 \mathrm{~m}^{3} \cdot \mathrm{s}^{-1}, \alpha_{n=7}=\dot{V}_{\text {rr } n=7} / \dot{V}_{\text {rrs }}=89.31 \%$. The error is over $10 \%$.

2. Using minimization results $v_{\mathrm{Cm}}=5.866 \mathrm{~m} \cdot \mathrm{s}^{-1}, n=11.049$, and same constructional parameters we obtain

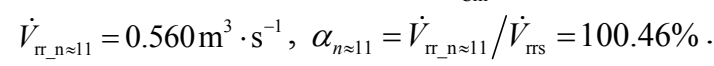

The error is very small, however, this method is very difficult in practical measurement implementation. The measured velocity distribution profile in cross section is required, exactly along the short axis. Measurement the profile inside the duct is practically impossible.

3. It is possible to estimate the parameter on the basis of two measurements (readings): at points B and C - see Fig. 1. The velocity $v_{\mathrm{B}}$ was read (measured) for $h=0.25 \mathrm{H}$. Therefore the expression for $n$ is

$$
v_{\mathrm{B}}=v_{\mathrm{C}}\left(1-\frac{0.25 H}{0.5 H}\right)^{\frac{1}{n}}=v_{\mathrm{C}} 0.5^{\frac{1}{n}} \Rightarrow n=\frac{\ln (0.5)}{\ln \left(v_{\mathrm{B}}\right)-\ln \left(v_{\mathrm{C}}\right)}
$$

Using of two-point velocity measurement: $v_{\mathrm{C}}=v_{\mathrm{C} 16}=5,678 \mathrm{~m} \cdot \mathrm{s}^{-1}$ and $v_{\mathrm{B}}=5,332 \mathrm{~m} \cdot \mathrm{s}^{-1}$. Calculated on the basis of formula (5), the value of $n$ is $n=11.012$, so, after substituting to formula (1) values of $n, v_{\mathrm{C} 16}, \Lambda, \mathrm{P}, W$ we obtain $\dot{V}_{\mathrm{tp}}=0.541 \mathrm{~m}^{3} \cdot \mathrm{s}^{-1}$, therefore, the quality indicator of the estimate $\alpha_{\mathrm{tp}}=\dot{V}_{\mathrm{tp}} / \dot{V}_{\mathrm{rrs}}=97.19 \%$. The disadvantage of this method is primarily the high sensitivity of the logarithmic function to the measurement accuracy (reading). 


\subsection{Basic power-law velocity profile in slot-rounded square cross-section}
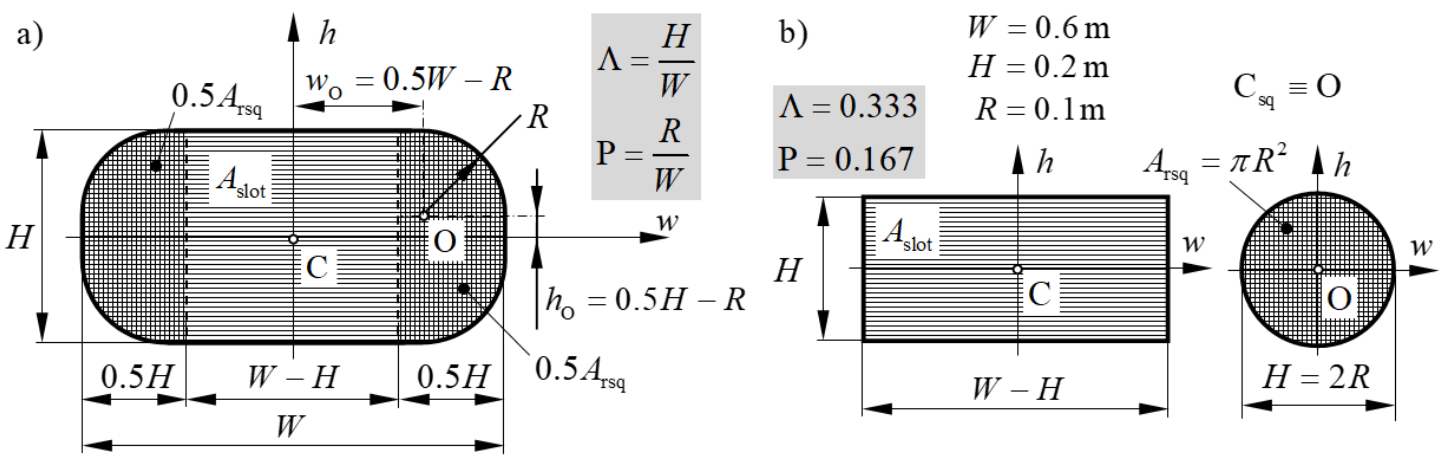

Fig. 5: Division of the cross-section area for the rectangle model - rounded square: a) general view; b) cross-section analyzed.

In this new model, it has been assumed that the flow in the duct has a twofold character: one-dimensional as in the slot and two-dimensional as in the rounded square cross-section (Fig. 5). Average velocity is determined by formula in (Peszyński et al., 2017). In the case under consideration $2 \mathrm{P} / \Lambda=1$, therefore the formula (6) is reduced to the form

$$
\dot{V}_{\text {srsq }}=\dot{V}_{\text {slot }}+\dot{V}_{\text {cir }}=\frac{n}{n+1}(1-\Lambda) \Lambda W^{2} v_{\mathrm{C}}+\pi \frac{2 n^{2} \mathrm{P}^{2}}{(2 n+1)(n+1)} W^{2} v_{\mathrm{C}}=\frac{n}{n+1}\left((1-\Lambda) \Lambda+\pi \frac{2 n \mathrm{P}^{2}}{2 n+1}\right) W^{2} v_{\mathrm{C}}
$$

1. For $v_{\mathrm{C} 16}=5,678 \mathrm{~m} \cdot \mathrm{s}^{-1}, n=7, \Lambda=0.333, \quad \mathrm{P}=0.167$, and $W=0.6 \mathrm{~m}$ we obtain from formula

$\dot{V}_{\text {slot }}=0.397 \mathrm{~m}^{3} \cdot \mathrm{s}^{-1}, \dot{V}_{\text {cir }}=0.146 \mathrm{~m}^{3} \cdot \mathrm{s}^{-1}$, therefore $\dot{V}_{\text {sssq }}=0.543 \mathrm{~m}^{3} \cdot \mathrm{s}^{-1}$ and $\alpha_{n=7}=\dot{V}_{\text {ssq } \mathrm{n}=7} / \dot{V}_{\text {rss }}=97.50 \%$.

2. Using minimization results $v_{\mathrm{Cm}}=5,866 \mathrm{~m} \cdot \mathrm{s}^{-1}, n=11.049$, and same constructional parameters we obtain $\dot{V}_{\text {ssas_ } \_\approx 11}=0.592 \mathrm{~m}^{3} \cdot \mathrm{s}^{-1}, \alpha_{n \approx 11}=\dot{V}_{\text {srqs_n } \approx 11} / \dot{V}_{\text {rrs }}=106.27 \%$.

3. Using $n=11.012$ from two-point velocity measurement, $v_{\mathrm{C} 16}=5,678 \mathrm{~m} \cdot \mathrm{s}^{-1}$, and same constructional parameters we obtain $\dot{V}_{\text {sras tp }}=0.573 \mathrm{~m}^{3} \cdot \mathrm{s}^{-1}$, therefore, the quality indicator of the estimate $\alpha_{\mathrm{tp}}=\dot{V}_{\mathrm{sus} \leq \mathrm{p}} / \dot{V}_{\mathrm{rrs}}=102.83 \%$.

\section{Conclusions}

Numerical simulation generally confirmed the assumptions regarding the development of velocity profile along the duct axis and the assumption regarding the length of the duct necessary to stabilize this profile.

The analysis of individual models showed that the derived formulas can be used to determine the volumetric flow rate, while it did not give an unequivocal answer to the question which one is the best. It is difficult to make such a decision on the basis of tests of one section. It should be remembered that the ventilation system being developed comprised 79 cross-sections.

\section{References}

Abe, H., Kawamura, H., Matsuo, Y. (2001) Direct Numerical Simulation of a Fully Developed Turbulent Channel Flow With Respect to the Reynolds Number Dependence. J. Fluids Eng., 382.

Peszyński, K. (2019) Linear Pressure Losses Coefficients in a New Ventilation System in Proc. $25^{\text {th }}$ Int. Conf. on Engineering Mechanics, (eds. Zolotarev I., and Radolf V.) Svratka, Czech Republic, pp. 279-282.

Peszyński, K. (2018) Building a bridge between industry and theory on the example of a new ventilation system. International Conference Experimental Fluid Mechanics 2018, pp. 9-14.

Peszyński, K., Novosád, J., Smyk, E, Olszewski, Ł., Dančová, P. (2017) Modelling of air flow rate in significantly flattened rounded rectangular ventilation ducts., EPJ Web of Conferences 180, 02082.

Smyk, E., Mrozik, D., Olszewski, Ł., Peszyński, K. (2017) Numerical simulation of minor losses coefficient on the example of elbows, EPJ Web of Conferences 180, 02093. 